\title{
Effect of Video Modeling Process on Teaching/Learning Hurdle Clearance Situations on Physical Education Students
}

\author{
Samiha Amara1, Bessem Mkaouer'1, Sarra H. Nassib', Helmi Chaaben², Younes Hachana1, \\ Fatma Z. Ben Salah ${ }^{3}$ \\ ${ }^{1}$ Higher Institute of Sport and Physical Education of Ksar Saïd, University of Manouba, Manouba, Tunisia \\ ${ }^{2}$ National Centre of Medicine and Sport Science of Tunis, Tunis, Tunisia \\ ${ }^{3}$ Higher Institute of Special Education, University of Manouba, Manouba, Tunisia \\ Email: samiha_ath@yahoo.fr
}

Received 1 September 2015; accepted 2 November 2015; published 5 November 2015

Copyright (C) 2015 by authors and Scientific Research Publishing Inc.

This work is licensed under the Creative Commons Attribution International License (CC BY). http://creativecommons.org/licenses/by/4.0/

(c) (i) Open Access

\begin{abstract}
The purpose of this study was to investigate the difference between two pedagogical methods in teaching/learning hurdle clearance such as the traditional learning method which was based on verbal feedback and the new technology through motion analysis and video modeling which was based on self-modeling, expert modeling and model's superposition. Twenty-seven sports science students took part in this study. Modeling group composed of 15 students and traditional group of 12 students. Both groups participated in a 10-week hurdle clearance learning (two sessions per week) which was composed of 10 sets (5 hurdles clearance per set) in total 1000 repetitions. The findings indicated a better enhancement of the learning score in the modeling group compared to the traditional one $(14.26 \pm 2.05$ pts vs. $11.66 \pm 1.72$ pts, respectively with $p<0.01)$. In addition, the analysis of delta-percentage revealed a high increase of technical performance in the modeling group. In conclusion, video feedback with model's superposition led to a better improvement of learning in hurdle clearance more than verbal feedback.
\end{abstract}

\section{Keywords}

Physical Education, Learning, Hurdle, Modeling, Video Feedback, Model's Superposition

\section{Introduction}

The modeling of human body's simulations is the subject of many studies in sports field, due to the evolution of

How to cite this paper: Amara, S., Mkaouer, B., Nassib, S. H., Chaaben, H., Hachana, Y., \& Salah, F. Z. B. (2015). Effect of Video Modeling Process on Teaching/Learning Hurdle Clearance Situations on Physical Education Students. Advances in Physical Education, 5, 225-233. http://dx.doi.org/10.4236/ape.2015.54027 
information and communication technology (ICT) as well as the appropriate software in the recent decades (Casey \& Jones, 2012). Actually, the biomechanical human models described the movement, as far as possible, to define its differentiation with changes in biomechanical characteristics (Ballreich, 1980). Thus, modeling the athletic movement is a functional representation of the body in motion to create artificially one or more variables (technical), derived from a theoretical approach (strength, speed, momentum...) to replace complex patterns by simplified ones (Laffay \& Orsay, 2008). However, the model presents a powerful catalyst in motor skill learning that helps so much with the process of teaching or training (Kalapoda, Michalopoulou, Aggelousis, \& Taxildaris, 2003).

The literature reveals a multitude of video feedback forms underlying the modeling method. More recently, three modeling forms have been presented: 1) self-modeling is a form of observational learning with the distinction that the observed and the observer, object, and subject, are the same person, 2) expert-modeling where the observed and the observers are not the same person and 3) model's superposition [self vs. expert model], (Boyer et al. 2009; Dowrick, 2000, 2012).

Adashevskiy, Iermakov, Korzh, Muszkieta, Krzysztof, \& Cieślicka (2014), Harvey \& Gittins (2014), Trout (2013), Stoicescu \& Stanescu (2012), Wilson (2008), Tofan, Vlase, Teodorescu, Burca, \& Candea (2006) and Durey (1995) noted that the modeling of sports skills, through simulation software and motion analysis (i.e., Human Motion Bulider, SkillSpector and Kinovea) developed athletes’ knowledge and motor performance. Bandura (1997) showed the role of modeling in enhancing skill acquisition. Reo \& Mercer (2004) demonstrated that videotaped modeling is more effective than traditional learning to achieve performance accuracy of a basic exercise program. In accordance with Herbert and Landin (1994), the learning by video model can facilitate learning by involving the student in problem-solving skills regarding error correction.

Interestingly, several studies showed a beneficial effect of video feedback in different physical activities (Atienza, Balaguer, \& Garcia-Merita, 1998; Baudry, Leroy, \& Chollet, 2006; Boyer, Miltenberger, Batsche, \& Fogel, 2009; Clark \& Ste-Marie, 2007; Eery \& Morizot, 2000; Guadagnoli \& Holcomb, 2002; Herbert \& Landin, 1994; Hodges, Chua, \& Franks, 2003; Laguna, 2000; Magill \& Schoenfelder-Zohdi, 1996; Parsons \& Alexander, 2012; Weir \& Connor, 2009; Wulf, 2007; Wulf \& Prinz, 2001), however, until now only three previous researchers have examined the athletic events. Cheraghidocheshmeh, Darush, \& Mojtaba (2009) presented an important impact of educational technology on learning in exercise technical skills in amateur athletes. Specifically, Giroud \& Debû (2004) and Palao et al. (2013) showed the video feedback efficiency for hurdle clearance learning.

These researches established in hurdle clearance (i.e., Giroud \& Debû, 2004; Palao et al. 2013) have used only self-modeling and expert modeling with a real-time demonstration or video-scope model, the information is issued identically repeated during learning sequence. These studies involve quantitative and qualitative measures of knowledge and skill of student practice. By consequence, the purpose of this study was to introduce the model's superposition (self-modeling vs expert-modeling) in video feedback learning and to use qualitative and quantitative measure to compare whether learning video feedback was relative to verbal feedback. In fact, this might help to determine the extent of deep learning achieved by physical education students engaged with ICT in teaching/learning hurdle clearance.

\section{Methods}

\subsection{Participants}

Twenty-seven sports science students in the Higher Institute of Sport and Physical Education, spread accordingly over two classes of second year degree in Physical Education [Class (A) composed of 15 students (age, 21.41 \pm 1.23 years; height, $1.79 \pm 0.06 \mathrm{~m}$; mass, $74.1 \pm 8.3 \mathrm{~kg}$ ); Class (B) composed of 12 students (age, $20.94 \pm 2.51$ years; height, $1.80 \pm 0.04 \mathrm{~m}$; mass, $75.3 \pm 6.7 \mathrm{~kg}$ )] and one-expert hurdle athlete (Tunisian and Arabic champion 2012, age, 21.45 years; height, $1.87 \mathrm{~m}$; mass, $80.2 \mathrm{~kg}$ ) took part in this study. After being informed in advance about the procedures, methods, benefits and possible risks involved in the study, a written consent must be obtained from participants. The experimental protocol was performed in accordance with the Declaration of Helsinki for human experimentation and was approved by the Ethical Committee.

\subsection{Acquisition Process of the Athlete's Model}

A technical model for an expert hurdle race's athlete was established in the fourth and fifth hurdle (Coh, 2003; 
Coh, Zvan, \& Jost, 2004) and adapted from the stride model presented by Hay (1980). The collected clearance kinematic data from SkillSpector software was used to determine technical model (Mkaouer, Jemni, Amara, Chaabène, \& Tabka 2013) (Figure 1).

\subsection{Experimental Design and Procedures}

This study examined the effect of video modeling on learning hurdle race's situation. Participants were spread over two classes, one of which participated in the video modeling learning (Class A or "modeling group") and the other one followed a traditional learning (Class B or "traditional group"). The study followed a pretest/posttest experimental design, taken before and after measurements of each group, in order to explore the effects of the learning method, in which students have been participated (modeling or classical), on students' achievement.

The two classes had the same structure: warm-up, initial information, main part, and a lesson summary by the teacher using video feedback or/and verbal feedback. Both groups participated in a 10-week hurdle clearance learning cycle, during track and field athletics sessions (two session's per-week of 90 minutes) composed by 10 sets (5 hurdles clearance per set, in total 1000 repetitions) in the academic year 2014/2015.

In classes, the initial information was given by the teacher, and involved the explanation of the class objectives, the tasks, and the key technical aspects of the lesson. The same verbal instructions for development of skills and knowledge in different phase of hurdles clearance (i.e., takeoff, flight and landing) was unified for both groups (Ericsson \& Simon, 1993).

Takeoff phase. To establish a trajectory which minimizes the height over the hurdle, the center of mass (COM) should be raised only as high as needed for the athlete to clear the hurdle efficiently (Rogers, 2000). High body position is essential for the attack. Drive is more forward than upward (Run "into" the hurdle, do not jump). The hip, knee and ankle joints of the support leg are fully extended. And Thigh of the lead leg swings rapidly to the horizontal position (Rogers, 2000).

Flight phase. To minimize the loss of speed and time in the air: takeoff is well in front of the hurdle from the ball of the foot (two thirds of the overall hurdle stride), (Coh, 2003). Lead leg is actively lowered as quickly as possible after the hurdle. Lower part of the lead leg is actively extended forwards in the direction of running, foot of the lead leg is flexed, trunk is well bent for higher hurdles, bending is less exaggerated for lower hurdles and shoulders remain parallel to the hurdle. Trail leg is drawn alongside the body, thigh of the trail leg is roughly parallel to the ground at clearance. Angle between the thigh and lower leg is about $90^{\circ}$, ankle of the trail leg is flexed markedly, toe is tilted upward and the knee of the trail leg is kept high as it pulls through (Rogers, 2000). Minimize the height of the COM above the hurdle (Coh, 2003).

Landing phase. Stay tall, to make a fast transition to running: landing leg is rigid and active on the ball of the foot (no heel contact at touchdown), body should not lean backwards on landing, trail leg stays tucked until touchdown then it pulls quickly and actively forwards and contact with the ground is brief, the first stride is aggressive (Rogers, 2000).

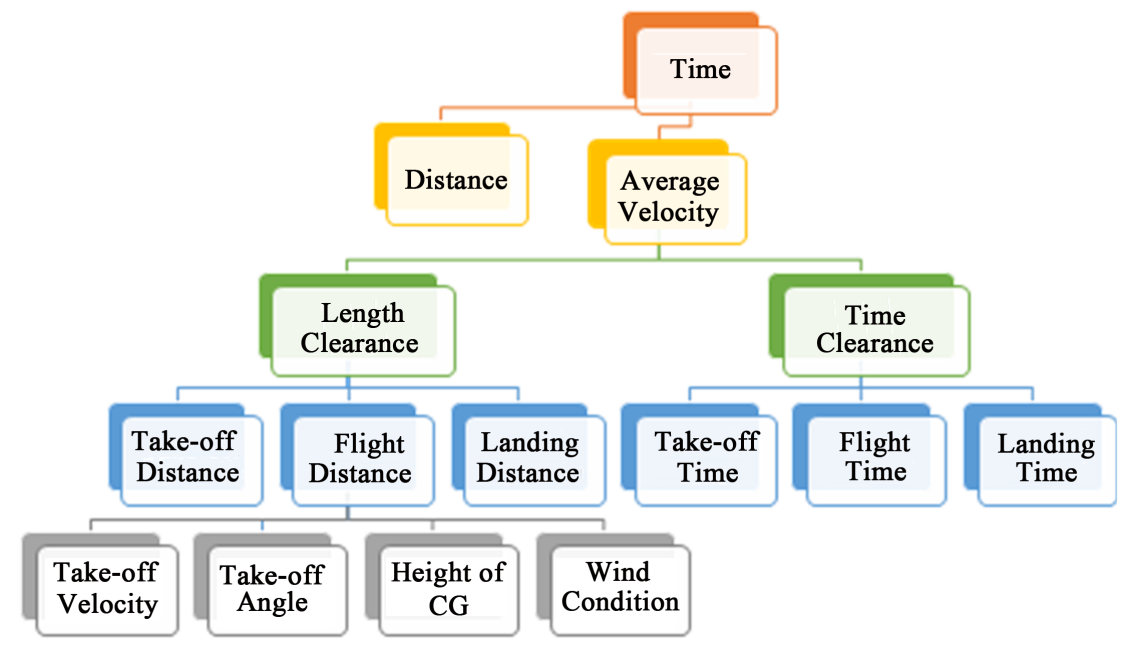

Figure 1. Technical model of hurdle clearance. 
Feedback and verbal instructions was given progressively according to students evolution and faults committed.

\subsubsection{Traditional Group}

This group, which is founded on teach/learn process with verbal instructions, based on teacher's interventions (i.e., verbal instruction and demonstration), technical faults corrections (i.e., in takeoff, flight and landing) and knowledge of hurdle clearance (i.e., angle of attack, body inclination, lead leg and trial leg actions) after each set. It is a classical learning without any video support.

\subsubsection{Modeling Group}

This group, which is founded on teach/learn process with video-feedback, using DartFish ${ }^{\circledR}$ software (Andrews \& Bobo, 2010; Harris, 2009; Uhl \& Dillon, 2009) in addition to verbal instruction. The processes of learning consist of the use of video feedback with self-modeling, expert-modeling and model's superposition to correct technical faults. Different visualization was used (i.e., technical analyze, self-modeling, expert-modeling and model's superposition self vs. expert model) in real cadence (2-times) and with slow motion (1-time) after each set (Figure 2).

\subsubsection{Evaluation Process}

A scorecard was established for assessing hurdle clearance technique before and after the learning/teaching programs with a maximal score of 20 points for the ten technical variables studied [i.e., very good (2 pts); good (1.5 pts); medium (1 pts); weak ( 0.5 pts) for each variable] based on kinematic model data (Table 1$)$. We used the Dartfish $^{\circledR}$ software for assessing hurdle clearance technique before and after the learning/teaching programs for the two groups (Andrews \& Bobo, 2010; Harris, 2009; Uhl \& Dillon, 2009).

\subsection{Statistical Analysis}

Data are reported as mean \pm standard deviation (SD). Effect size (dz) was calculated using GPOWER software (Bonn FRG, Bonn University, Department of Psychology), (Faul \& Erdfelder 2004). The following scale was used to interpret the dz: $<0.2$, [trivial]; $0.2-0.6$, [small]; 0.6 - 1.2, [moderate]; $1.2-2.0$, [large]; and $>2.0$, [very large], (Hopkins, 2002; Scanlan, Dascombe, \& Reaburn 2012). All variables were tested for normality by the Shapiro-Wilk test and results showed that the data were normally distributed. Therefore, the Student's t-test was

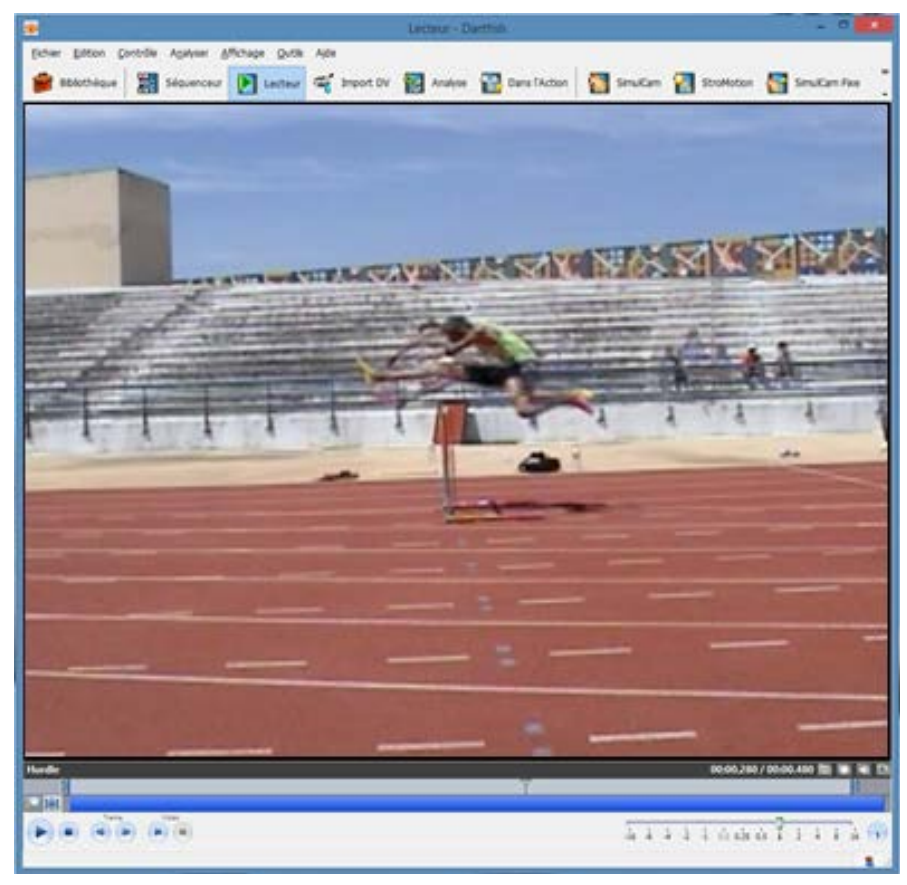

Figure 2. Video-feedback of self vs. expert model with Dartfish. 
Table 1. Criteria of assessment hurdle clearance.

\begin{tabular}{|c|c|c|c|c|c|c|c|c|c|c|}
\hline & \multicolumn{4}{|c|}{ Takeoff phase } & \multicolumn{2}{|c|}{ Flight phase } & \multicolumn{4}{|c|}{ Landing phase } \\
\hline & $\begin{array}{l}\text { Takeoff } \\
\text { angle }\left({ }^{\circ}\right)\end{array}$ & $\begin{array}{c}\text { Takeoff } \\
\text { distance (m) }\end{array}$ & $\begin{array}{c}\text { Takeoff } \\
\text { time (sec) }\end{array}$ & $\begin{array}{c}\text { High of } \\
\text { COM (m) }\end{array}$ & $\begin{array}{l}\text { Flight time } \\
\text { (sec) }\end{array}$ & $\begin{array}{l}\text { High of } \\
\text { COM (m) }\end{array}$ & $\begin{array}{l}\text { Landing } \\
\text { angle }\left({ }^{\circ}\right)\end{array}$ & $\begin{array}{c}\text { Landing } \\
\text { distance (m) }\end{array}$ & $\begin{array}{l}\text { Landing } \\
\text { time (sec) }\end{array}$ & $\begin{array}{l}\text { High of } \\
\text { COM (m) }\end{array}$ \\
\hline $\begin{array}{l}\text { Very good } \\
\text { (2 pts) }\end{array}$ & $72-74$ & $1.91-2.00$ & 0.100 & $1.10-1.13$ & $0.34-0.36$ & $0.45-0.47$ & $78-80$ & $1.51-1.60$ & 0.080 & $1.22-1.25$ \\
\hline $\begin{array}{c}\text { Good } \\
\text { (1.5 pts) }\end{array}$ & $69-71$ & $1.81-1.90$ & 0.120 & $1.14-1.17$ & $0.37-0.39$ & $0.48-0.50$ & $75-77$ & $1.41-1.50$ & 0.100 & $1.18-1.21$ \\
\hline $\begin{array}{l}\text { Medium } \\
\text { (1 pt) }\end{array}$ & $66-68$ & $1.71-1.80$ & 0.140 & $1.18-1.21$ & $\begin{array}{l}0.40 \\
0.42\end{array}$ & $0.51-0.53$ & $72-74$ & $1.31-1.40$ & 0.120 & $1.14-1.17$ \\
\hline $\begin{array}{l}\text { Weak } \\
(0.5 \text { pts })\end{array}$ & $63-65$ & $1.61-1.70$ & 0.160 & $1.22-1.25$ & $0.43-0.45$ & $0.54-0.56$ & $69-71$ & $1.21-1.30$ & 0.140 & $1.10-1.13$ \\
\hline
\end{tabular}

(COM) Center of mass.

applied to compare the difference between the test and the retest (paired t-test) and between groups (unpaired t-test). The delta-percentage $\left(\Delta_{\%}\right)$ between the test and the retest $\left[\Delta_{\%}=((\right.$ retest - test $) /$ test $\left.) \times 100\right]$ was determined. Significance level was set at $0.05 \%(p<0.05)$. Calculations and statistical analyses were performed using Statistical Package for the Social Sciences version 20.0 (SPSS Inc., Chicago, IL, USA).

\section{Results}

Within-group analysis showed that both groups have improved their hurdle clearance technique (9.33 \pm 1.92 pts vs. $14.26 \pm 2.05$ pts and $9.13 \pm 1.67$ vs. $11.67 \pm 1.72$ pts, respectively for modeling group and traditional group with $p<0.01$ ), but modeling group had a higher mean technique score, $26.88 \%$ of increase more than the traditional group (55.81\% vs. 28.93\%, respectively modeling group and traditional group).

Between-group analysis revealed no significant difference in the test among modeling group and traditional group (9.33 \pm 1.92 pts vs. $9.13 \pm 1.67$ pts, respectively with $p>0.05)$. Moreover, a significant difference were registered between modeling group and traditional group in the retest $(14.27 \pm 2.05$ pts vs. $11.67 \pm 1.72$ pts, respectively with $p<0.01)$ and in the percentage of improvement "delta-percentage" ( $\Delta_{\%}=55.81 \% \pm 27.77 \%$ vs. $28.93 \% \pm 10.04 \%$, respectively with $p<0.01$ ) (Table 2; Figure 3 ).

\section{Discussion}

This study is a comparative investigation of two different learning methods. The first one is a video feedback learning combining expert-modeling, self-modeling and model's superposition (modeling group). The second one is a classical learning based on verbal feedback, teacher's interventions, demonstrations and knowledge of performance (traditional group). Obviously, the most important finding of this investigation is the benefit of video-feedback with model's superposition, which allows a better improvement in the technical learning. The results indicated a significant difference $(p<0.01)$ between the modeling group and the traditional one on learning scores and delta-percentage (test vs. retest) across a 10-week period. However, both methods showed a significant learning improvement $(p<0.01)$. The traditional group, with verbal feedback, enhances the acquisition of appropriate movement patterns, which subsequently leads to better outcomes (Hebert \& Landin, 1994). Moreover, this group, where the video feedback (i.e., expert-modeling, self-modeling and model's superposition) was not presented, can have information in the performance of their peers performing the same training sequences "inexperienced-modeling”. Otherwise, the modeling group has better improved its technical performances compared to the traditional one $\left(\Delta_{\%}=55.81 \% \pm 27.77 \%\right.$ and $28.93 \% \pm 10.04 \%$, respectively with $p<$ 0.01). This enhancement can be allocated to the different forms of video feedback (i.e., expert-modeling, self-modeling and model's superposition), that provide a significant information to optimize the performance (Baudry et al. 2006; Giroud \& Debû, 2004; Palao et al. 2013).

In the field of motor skills, this finding has been validated as well, particularly by the study of Carroll \& Bandura (1987). The authors prove that the observation of an external model is effective at the beginning of learning for the acquisition of a new coordination consists of a complex motor sequence. In a second phase, knowledge of performance, delivered through a video feedback, enables the refinement of the internal model. 


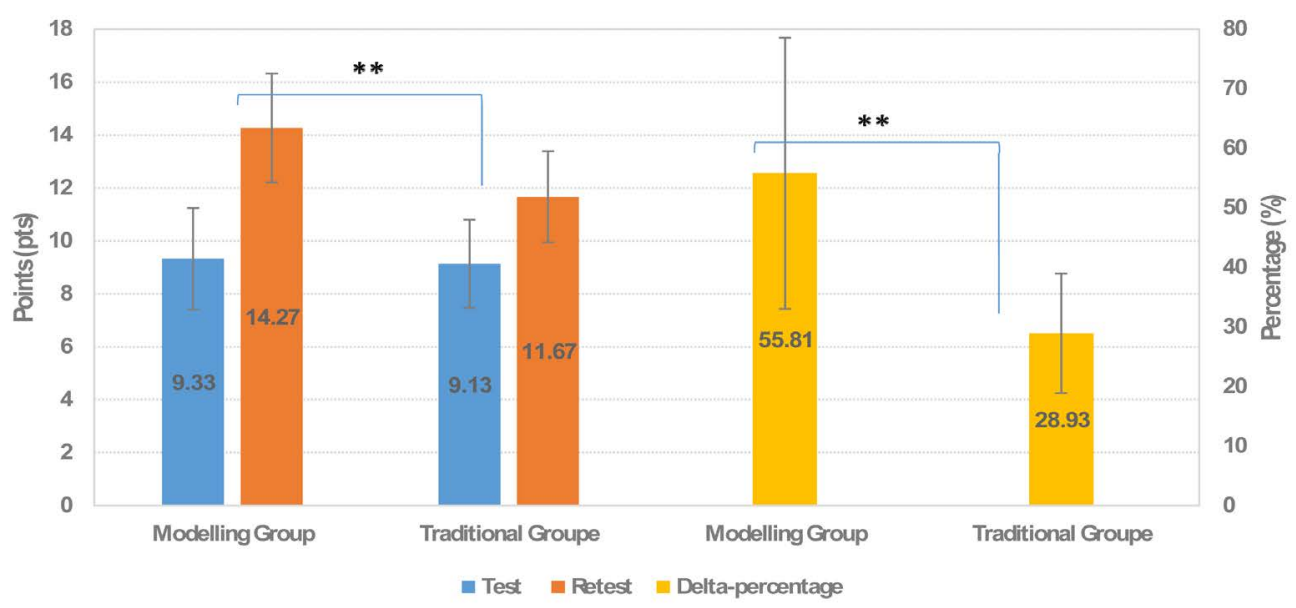

Figure 3. Technical score and delta-percentage before and after learning programs.

Table 2. Unpaired Student's t-test, modeling group versus traditional group.

\begin{tabular}{ccccc}
\hline Variables & $\mathrm{t}$ & $\mathrm{df}$ & Sig. & Effect size $(\mathrm{dz})$ \\
\hline Test & 0.226 & 25 & 0.769 & 0.117 \\
Retest & $3.507^{*}$ & 25 & 0.002 & $1.374^{+}$ \\
Delta-percentage & $3.794^{*}$ & 25 & 0.001 & $1.528^{+}$ \\
\hline
\end{tabular}

( ) Significant at $p<0.01$; $\left(^{+}\right)$Moderate Effect Size.

The video observation of a reference model, in this case is an elite athlete, is interesting for several reasons. Indeed, when the model is an expert athlete, the convenient skill execution on performance will serve as a source of information for observers (Wulf, 2007; Wulf \& Prinz, 2001). In this context, the expert-model provides the learner with information to reach the task goal (Magill \& Schoenfelder-Zohdi, 1996; Wulf, 2007; Wulf \& Prinz, 2001). A visual model may serve as the best mechanism for encoding and retrieving information from memory (Meany, 1994). The repeated observation of a reference model would facilitate the development of internal reference (Magill \& Schoenfelder-Zohdi, 1996) or cognitive representation (Carroll \& Bandura, 1985, 1990), necessary for appropriation of essential coordination to perform a complex motor skill. In addition, the information obtained by self-modeling, can influence the development of a memory representation of the skill and facilitate technical faults correction and subsequent learning (Giroud \& Debû, 2004; Kalapoda et al. 2003; Palao et al. 2013).

Finally, the modeling form has proven effective in promoting student learning hurdle race. So, the technical score of modeling group is significantly higher than the traditional one $(14.27 \pm 2.05$ pts and $11.67 \pm 1.72$ pts, respectively with $p<0.01$ ). Learners observe the expert-model, the self-model and model's superposition then rehearse and retain the skill information, which is used to reproduce or correct the movement, have improved their technical abilities compared to learners with verbal feedback (Clark \& Ste-Marie, 2007; Giroud \& Debû, 2004; Kalapoda et al. 2003; Palao et al. 2013). These results add another extent for the studies of Giroud \& Debû (2004) and Palao et al. (2013), the deep learning with a video feedback using model's superposition is better than verbal feedback in teaching/learning hurdle clearance. Accordingly, using the model's superposition, it provides educators with pedagogical tools to promote a deeper understanding of the human movement, its relationship with athletic performance and practical application of ideas and knowledge.

\section{Conclusion}

Overall, both verbal feedback and video feedback lead to improving learning and performance in hurdle clearance but the range of improving in video feedback group is better than in verbal feedback group. Our study provides evidence that supports an increased role of ICT for education on learning in exercise technical skills for physical education students, which allows for the educators and students to find the right answer or solution to a similar problem or situation. Therefore, it is essential for educators to incorporate video learning activities (i.e., 
self-modeling, expert-modeling and model's superposition) during students training programs to integrate opportunities for students to gain the specific knowledge through video modeling related to which the human body provides a link to the specific domains of athletic training. Frequently, to address the educational competencies directly associated with modeling process, many programs require a strong background for hurdle technical skills. Consequently, the current findings have important practical implications for students, athletes and coaches who can benefit from the importance of model's superposition process in athletic performance.

\section{Acknowledgements}

We would like to thank all students as well as the ISSEP teachers for their valuable help and invaluable common sense while dealing with this study.

\section{References}

Adashevskiy, V. M., Iermakov, S. S., Korzh, N. V., Muszkieta, R., Krzysztof, P., \& Cieślicka, M. (2014). Biomechanical Study Athletes' Movement Techniques in the Hurdles (on Example of Phase of Flight). Physical Education Student, 4, 3-12.

Andrews, A., \& Bobo, L. (2010). Performance Measurement and Assessment Using Dartfish Software. In J. Sanchez, \& K. Zhang (Eds.), Proceedings of E-Learn: World Conference on E-Learning in Corporate, Government, Healthcare, and Higher Education 2010 (pp. 407-408). Orlando: Association for the Advancement of Computing in Education.

Atienza, F.L., Balaguer, I., \& Garcia-Merita, M. L. (1998). Video Modeling and Imaging Training on Performance of Tennis Service of 9- to 12-year-old Children. Perceptual and Motor Skills, 87, 519-529.

http://dx.doi.org/10.2466/pms.1998.87.2.519

Ballreich, R. (1980). Aspekte der Modellierung in der Biomechanik des Sports [Aspects of Modeling in Biomechanics of Sport]. In W. Baumann (Ed.), Biomechanik und sportliche Leistung (pp. 113-128). Schorndorf: Hofmann.

Bandura, A. (1997). Self-efficacy: The exercise of control. Freeman: New York.

Baudry, L., Leroy, D., \& Chollet, D. (2006). The Effect of Combined Self- and Expert-Modeling on the Performance of the Double Leg Circle on the Pommel Horse. Journal of Sports Sciences, 24, 1055-1063. http://dx.doi.org/10.1080/02640410500432243

Boyer, E., Miltenberger, R. G., Batsche, C., \& Fogel, V. (2009). Video Modeling by Experts with Video Feedback to Enhance Gymnastics Skills. Journal of Applied Behavior Analysis, 42, 855. http://dx.doi.org/10.1901/jaba.2009.42-855

Carroll, W. R., \& Bandura, A. (1985). Role of Timing of Visual Monitoring and Motor Rehearsal in Observational Learning of Action Patterns. Journal of Motor Behavior, 17, 269-281. http://dx.doi.org/10.1080/00222895.1985.10735349

Carroll, W. R., \& Bandura, A. (1987). Translating Cognition into Action: The Role of Visual Guidance in Observational Learning of Action Patterns. Journal of Motor Behavior, 19, 153-167. http://dx.doi.org/10.1080/00222895.1987.10735419

Carroll, W. R., \& Bandura, A. (1990). Representational Guidance of Action Production in Observational Learning: A Causal Analysis. Journal of Motor Behavior, 22, 85-97. http://dx.doi.org/10.1080/00222895.1990.10735503

Casey, A., \& Jones, B. (2012). Using Digital Technology to Enhance Student Engagement in Physical Education. Asia-Pacific Journal of Health, Sport and Physical Education, 2, 51-66.

Cheraghidocheshmeh, M., Darush, N., \& Mojtaba, I. (2009). The Comparison of Effect of Video-Modeling and Verbal Instruction on the Performance in Throwing the Discus and Hammer. Procedia-Social and Behavioral Sciences, 1, 27822785. http://dx.doi.org/10.1016/j.sbspro.2009.01.493

Clark, S. E., \& Ste-Marie, D. M. (2007). The Impact of Self-as-a-Model Interventions on Children's Self-Regulation of Learning and Swimming Performance. Journal of Sports Sciences, 25, 577-586. http://dx.doi.org/10.1080/02640410600947090

Coh, M. (2003). Biomechanical Analysis of Colin Jackson's Hurdle Clearance Technique. New Studies in Athletics, 18, 3745.

Coh, M., Zvan, M., \& Jost, B. (2004). Kinematical Model of Hurdle Clearance Technique. Proceedings of the 22th International Symposium of Biomechanics in Sports, ISBS, Ottawa, 8-12 August 2004, 311-314.

Dowrick, P. W. (2000). A Review of Self-Modeling and Related Interventions. Applied and Preventive Psychology, 8, 23-39. http://dx.doi.org/10.1016/S0962-1849(99)80009-2

Dowrick, P. W. (2012). Self-Modeling: Expanding the Theories of Learning. Psychology in the Schools, 49, 30-41. http://dx.doi.org/10.1002/pits.20613

Durey, A. (1995). L’EPS et son rapport à la technique [The PE and Its Relationship with Technique]. Revue Spirales, 8, 93-99. 
Eery, Y. A., \& Morizot, P. (2000). Kinesthetic and Visual Image in Modeling Closed Motor Skills: The Example of the Tennis Serve. Perceptual and Motor Skills, 90, 707-722. http://dx.doi.org/10.2466/pms.2000.90.3.707

Ericsson, K. A., \& Simon, H. A. (1993). Protocol Analysis: Verbal Reports as Data. A Bradford Book, London: The MIT Press.

Faul, F., \& Erdfelder, E. (2004). G Power: A Priori, Post-Hoc, and Compromise Power Analyses for MS-DOS (Computer Program). Bonn: Department of Psychology, University of Bonn.

Giroud, P., \& Debû, B. (2004). Effectiveness of Explicit or Implicit Demonstrating for Learning Hurdling in Children Aged 7 to 10 Years. Movement and Sports Science, 51, 29-48.

Guadagnoli, M., Holcomb, W., \& Davis, M. (2002). The Efficacy of Video Feedback for Learning the Golf Swing. Journal of Sports Sciences, 20, 615-622. http://dx.doi.org/10.1080/026404102320183176

Harris, F. (2009). Visual Technology in Physical Education. Physical and Health Education Journal, 74, 24-25.

Harvey, S., \& Gittins, C. (2014). Effects of Integrating Video-Based Feedback into a Teaching Games for Understanding Soccer Unit. Agora Para la Educación Física y el Deporte, Agora for PE and Sport, 16, 271-290.

Hay, J. G. (1980). Biomécanique des techniques sportives [Biomechanics of Sport Techniques]. Paris: Vigot.

Hebert, E. P., \& Landin, D. (1994). Effects of a Learning Model and Augmented Feedback on Tennis Skill Acquisition. Research Quarterly for Exercise and Sport, 65, 250-257. http://dx.doi.org/10.1080/02701367.1994.10607626

Hodges, N. J., Chua, R., \& Franks, I. M. (2003). The Role of Video in Facilitating Perception and Action of a Novel Coordination Movement. Journal of Motor Behaviors, 35, 247-260. http://dx.doi.org/10.1080/00222890309602138

Hopkins, W. G. (2002). A Scale of Magnitudes for Effect Statistics. Internet Society of Sports Science. http://www.sportsci.org/resource/stats/index.html

Kalapoda, E., Michalopoulou, M., Aggelousis, N., \& Taxildaris, K. (2003). Discovery Learning and Modeling When Learning Skills in Tennis. Journal of Human Movement, 45, 433-448.

Laffay, G., \& Orsay, U. S. (2008). La modélisation biomécanique: Un outil didactique? [Biomechanical Modeling: An Educational Tool?]. Revue Impulsion, 15, 130-143.

Laguna, P. (2000). The Effect of Model Observation versus Physical Practice during Motor Skill Acquisition and Performance. Journal of Human Movement Science, 39, 171-191.

Magill, R. A., \& Schoenfelder-Zohdi, B. (1996). A Visual Model and Knowledge of Performance as Sources of Information for Learning a Rhythmic Gymnastics Skill. International Journal of Sports Psychology, 27, 7-22.

Meany, K. S. (1994). Developmental Modeling Effects on the Acquisition, Retention, and Transfer of a Novel Motor task. Research Quarterly for Exercise and Sport, 65, 31-39. http://dx.doi.org/10.1080/02701367.1994.10762205

Mkaouer, B., Jemni, M., Amara, S., Chaabène, M., \& Tabka, Z. (2013). Kinematic and Kinetic Analysis of Two Gymnastics Acrobatic Series to Performing the Backward Stretched Somersault. Journal of Human Kinetics, 37, 17-26. http://dx.doi.org/10.2478/hukin-2013-0021

Palao, J. M., Hastie, P. A., Cruz, P. G., \& Ortega, E. (2013). The Impact of Video Technology on Student Performance in Physical Education. Technology, Pedagogy and Education, 24, 51-63. http://dx.doi.org/10.1080/1475939X.2013.813404

Parsons, J. L., \& Alexander, M. J. (2012). Modifying Spike Jump Landing Biomechanics in Female Adolescent Volleyball Athletes Using Video and Verbal Feedback. The Journal of Strength \& Conditioning Research, 26, 1076-1084. http://dx.doi.org/10.1519/JSC.0b013e31822e5876

Reo, J. A., \& Mercer, V. S. (2004). Effects of Live, Videotaped, or Written Instruction on Learning an Upper-Extremity Exercise Program. Physical Therapy, 84, 622-633.

Rogers, J. L. (2000). USA Track \& Field Coaching Manual. Champaign, IL: Human Kinetics.

Scanlan, A.T., Dascombe, B., \& Reaburn, P. R. J. (2012). The Construct and Longitudinal Validity of the Basketball Exercise Simulation Test. The Journal of Strength \& Conditioning Research, 26, 523-530.

http://dx.doi.org/10.1519/JSC.0b013e318220dfc0

Stoicescu, M., \& Stanescu, M. (2012). New Competencies for Physical Education Teachers: Software for Movement Analysis. Proceedings of the 8th International Scientific Conference "eLearning and Software for Education" (eLSE), Bucharest, 26-27 April 2012, 561-565. www.ceeol.com

Tofan, M., Vlase, S., Teodorescu, H., Burca, I., \& Candea, I. (2006). Virtual Analysis of the Athletic Motion. Hurdles Race. Proceedings of the 2nd WSEAS International Conference on Applied and Theoretical Mechanics, Venice, 20-22 November 2006, 155-157.

Trout, J. (2013). Digital Movement Analysis in Physical Education. Journal of Physical Education, Recreation \& Dance, 84, 47-50. http://dx.doi.org/10.1080/07303084.2013.818394 
Uhl, B., \& Dillon, S. (2009). Dartfish Video Analysis in Secondary Physical Education: A Pilot Study. Poster Session at the American Alliance for Health, Physical Education, Recreation, and Dance Annual Convention, Tampa, 31 March-4 April, 2009, 383.

Weir, T., \& Connor, S. (2009). The Use of Digital Video in Physical Education. Technology, Pedagogy and Education, 18, 155-171. http://dx.doi.org/10.1080/14759390902992642

Wilson, B. D. (2008). Development in Video Technology for Coaching. Sports Technology, 1, 34-40. http://dx.doi.org/10.1002/jst.9

Wulf, G. (2007). Attention and Motor Skill Learning. Champaign, IL: Human Kinetics.

Wulf, G., \& Prinz, W. (2001). Directing Attention to Movement Effects Enhances Learning: A Review. Psychonomic Bulletin \& Review, 8, 648-660. http://dx.doi.org/10.3758/BF03196201 